\title{
Encephalitis Related to Human Parechovirus Type 3
}

\author{
Wataru Miura ${ }^{a}$, Emiko Momoki ${ }^{a}$, Tatsuo Fuchigami ${ }^{a}$, c, Yuki Kasuga ${ }^{a}$, Kaori Kimura ${ }^{a}$, \\ Kazumasa Fuwa ${ }^{a}$, Yukihiko Fujita ${ }^{a}$, Hiroshi Ushijima ${ }^{b}$, Ichiro Morioka ${ }^{a}$
}

\begin{abstract}
Human parechoviruses belong to the Picornaviridae family and are classified into 17 genotypes. Recently, it has been reported that human parechovirus genotype 3 is an important cause of severe infections, including sepsis-like illness and encephalitis, especially in newborns and infants younger than 3 months. There are also reports that human parechovirus genotype 3 infections with central nervous system symptoms lead to high rates of neurological sequelae and death. Here, we report a case of a previously healthy 1-month-old girl who developed encephalitis related to human parechovirus genotype 3 and had a favorable outcome. The patient presented septicemic symptoms, including fever, tachycardia, tachypnea with retractions and seizures without an elevated inflammatory response. She lacked cerebrospinal fluid pleocytosis but had hypercytokinemia. Brain magnetic resonance imaging and electroencephalography showed abnormal findings. Together, these findings strongly suggested acute encephalopathy. She underwent emergency intubation for respiratory failure and mechanical ventilation was started. Intravenous phenobarbital injection was performed to prevent convulsion. She was treated using intravenous immunoglobulin with methylprednisolone pulse therapy. As of 2 years after discharge, her growth development is equivalent to her age and she has had no clinical epileptic seizures. In this case, human parechovirus genotype 3 was detected from pharyngeal swabs, stool, cerebrospinal fluid and blood by polymerase chain reaction assay. The patient was diagnosed definitively with encephalitis related to human parechovirus genotype 3 . The symptoms that we observed should be considered for the differential diagnosis of human parechovirus infection. When a patient is suspected of having encephalitis related to human parechovirus, treatment including intravenous immunoglobulin and corticosteroid must be started as soon as possible to prevent neurodevelopmental sequelae.
\end{abstract}

Keywords: Human parechovirus; Encephalitis; Newborns and young infants; Hypercytokinemia; Intravenous immunoglobulin; Corticosteroid

Manuscript submitted May 13, 2019, accepted August 21, 2019

aDepartment of Pediatrics and Child Health, Nihon University School of Medicine, 30-1 Oyaguchi-Kamicho, Itabashi-ku, Tokyo 173-8610, Japan

${ }^{b}$ Division of Microbiology, Department of Pathology and Microbiology, Nihon University School of Medicine, 30-1 Oyaguchi-Kamicho, Itabashi-ku, Tokyo 173-8610, Japan

${ }^{\mathrm{c} C}$ Corresponding Author: Tatsuo Fuchigami, Department of Pediatrics and Child Health, Nihon University School of Medicine, 30-1 Oyaguchi-Kamicho, Itabashi-ku, Tokyo 173-8610, Japan. Email: fuchigami.tatsuo@nihon-u.ac.jp

doi: https://doi.org/10.14740/ijcp333

\section{Introduction}

Human parechoviruses (HPeVs) belong to the Picornaviridae family and are classified into 17 genotypes [1]. Recently, it has been reported that human parechovirus genotype 3 (HPeV3) is an important cause of severe infections, including sepsislike illness and encephalitis, especially in newborns and young infants. Furthermore, $\mathrm{HPeV} 3$ usually causes acute encephalitis with white matter lesions, leading to neurological sequelae and death $[2,3]$. Here, we report a case of an infant with HPeV3related encephalitis who had a favorable outcome.

\section{Case Report}

A previously healthy 1-month-old girl presented with a fever of $38.8{ }^{\circ} \mathrm{C}$. The patient did not have poor feeding, gastrointestinal symptoms, or respiratory symptoms but was admitted to our hospital for suspected sepsis. She was delivered at a gestational age of 39 weeks (birth weight: 2,674 g) by vaginal delivery without complications such as asphyxia and hyperbilirubinemia. The prenatal examination of the mother showed normal findings. All her newborn screening evaluations were normal. Her developmental and other medical history were unremarkable. She was being breastfed and had no history of receiving any vaccinations; she lived with her healthy parents and 2-year-old sister. Her sister attended a nursery school; however, she had no cold symptoms. Her family's medical history was also unremarkable. No one in her family had cold symptoms or any other illness prior to the patient's illness.

On admission, her height was $54 \mathrm{~cm}$ (51st percentile) and her weight was 3,960 g (50th percentile). She was alert but irritable, with a body temperature of $38.5^{\circ} \mathrm{C}$. Her heart rate was 190 beats/min (tachycardia) and her respiration rate was 40 breaths/min (tachypnea). No bulging of the fontanel or cranial nerve deficit was observed. Neurological examination showed normal results. No abnormality was recognized by auscultation of the chest. Abnormal findings of abdominal distention and reticulated cyanosis of the limbs were observed.

Laboratory blood examination showed a reduced leukocyte count of $3.5 \times 10^{9} / \mathrm{L}$, but C-reactive protein results were not elevated. Serum chemistry was normal. Pyuria was not observed in the urine, and rapid antigen tests for respiratory syncytial virus, human metapneumovirus and influenza type $\mathrm{A}$ and $\mathrm{B}$ viruses were also negative. Her chest radiograph was normal. Gastrointestinal gas dilation was recognized on an abdominal radiograph. 


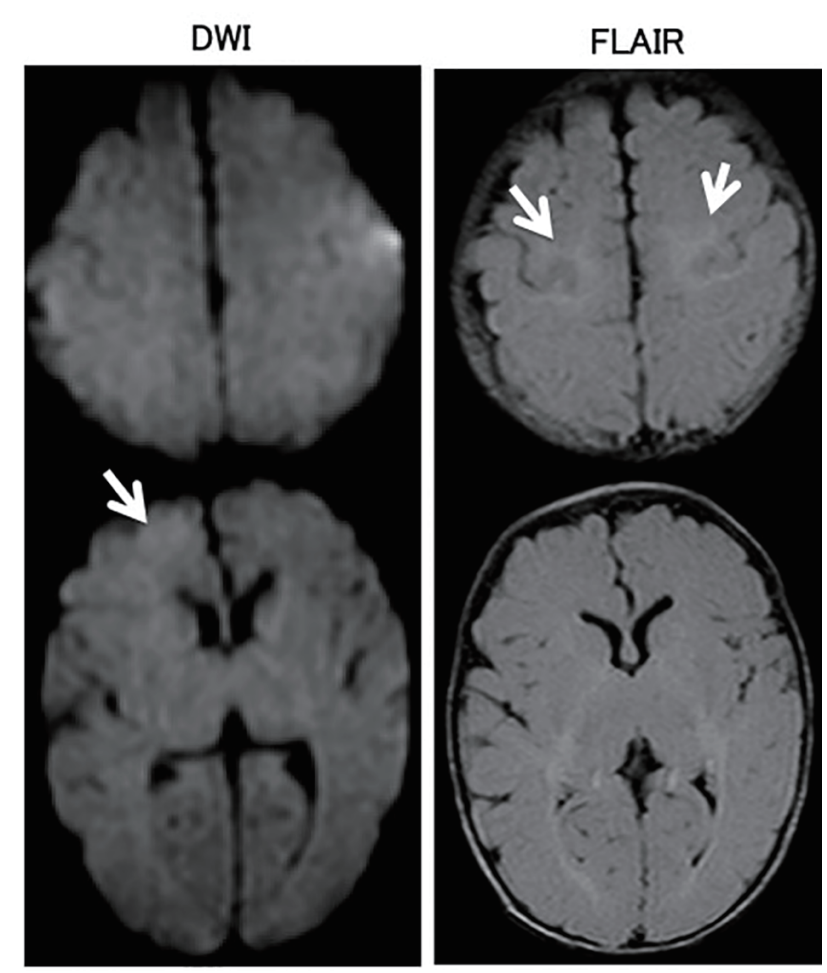

Figure 1. Brain magnetic resonance imaging on the third day of admission. In the brain magnetic resonance diffusion-weighted image (DWI), the right frontal lobe exhibited slightly high-intensity areas. T2-weighted fluid-attenuated inversion recovery (FLAIR) showed hyperintense lesions in bilateral parietal white matter.

On the second day after hospitalization, retraction breathing became evident in the evening. On the morning of the third day of admission, generalized clonic seizures of 5 min duration were recognized two times. In both cases, intravenous (IV) diazepam $(0.3 \mathrm{mg} / \mathrm{kg})$ was administered immediately and the seizures stopped. Brain computed tomography (CT), brain magnetic resonance (MR) imaging, electroencephalography and lumbar puncture were performed because of the appearance of seizures.

Laboratory blood examinations performed after the convulsions showed hyponatremia of $125 \mathrm{mmol} / \mathrm{L}$. In addition, elevated ferritin levels of $754 \mu \mathrm{g} / \mathrm{L}$ and high interleukin-6 (IL6) levels of $>109 \mathrm{pmol} / \mathrm{L}$ in plasma were observed using IL-6 STICKELISA ${ }^{\circledR}$ (Toray Medical Co., Ltd, Tokyo, Japan) [4], suggesting hypercytokinemia. Cerebrospinal fluid (CSF) analysis did not show any pleocytosis $(1 / \mu \mathrm{L})$, elevation in protein levels $(0.4 \mathrm{~g} / \mathrm{L})$, or decrease in glucose levels $(3.3 \mathrm{mmol} / \mathrm{L})$. Blood, urine and CSF bacterial cultures showed no growth. There was no obvious abnormality in cranial CT.

In brain MR diffusion-weighted image (DWI) on the third day of admission, the right frontal lobe exhibited slightly highintensity areas, and T2-weighted fluid-attenuated inversion recovery (FLAIR) showed hyperintense lesions in bilateral parietal white matter (Fig. 1). In an electroencephalogram (EEG) obtained on the third day of admission, spike waves were independently recognized in the left and right parietal regions. Slow waves were not observed in the background activity (Fig. 2).

These findings strongly suggested acute encephalopa- thy. Methylprednisolone pulse therapy $(30 \mathrm{mg} / \mathrm{kg} / \mathrm{day}$ for 3 days), $1 \mathrm{~g} / \mathrm{kg} /$ day of IV immunoglobulin (IVIG) for 2 days and edaravone $(1 \mathrm{mg} / \mathrm{kg} /$ day $)$ were promptly started. She underwent emergency intubation for respiratory failure on the same day, and mechanical ventilation was started. IV phenobarbital $(5 \mathrm{mg} / \mathrm{kg} /$ day $)$ injection was performed to prevent convulsion. Leukocytopenia, anemia and coagulopathy were complications, and daily administrations of ricomodulin and fresh-frozen plasma were necessary, along with transfusion of erythrocytes. Improvements in tachycardia and coagulopathy were observed after a first course of methylprednisolone pulse therapy. Her respiratory failure resolved, and she was taken off mechanical support on day 8 of hospitalization.

In brain MR imaging that was performed after two courses of methylprednisolone pulse therapy, the mild abnormal signal remained on FLAIR, but the findings were improved on DWI, and she was discharged without neurologic sequelae on the 18 th day of hospitalization. Her growth development is equivalent to her age as of 2 years after discharge. Although there have been no clinical epileptic seizures, epileptic discharges remain on her EEG findings, and anticonvulsive treatments with phenobarbital ( $5 \mathrm{mg} / \mathrm{kg} /$ day) was initiated to prevent further episodes until the age of 2 years. Currently, her age is 2 years and 5 months and she is receiving no medication.

$\mathrm{HPeV}$ infection was suspected because of the lack of specific clinical features, as well as the presence of hypercytokinemia. $\mathrm{HPeV} 3$ was detected from pharyngeal swabs, stool, cerebrospinal fluid and blood by polymerase chain reaction assay. She was diagnosed definitively with HPeV3-related encephalitis.

\section{Discussion}

$\mathrm{HPeV} 3$ causes severe disease in neonates and infants younger than 3 months, including sepsis-like symptoms and sepsis, meningitis and encephalitis [1]. Typically, young infants present with fever, tachycardia (often $>200$ beats $/ \mathrm{min}$ ), poor activity and poor feeding [1]. Clinical signs include mottled skin, abdominal distention, umbilical protrusion and palmar-plantar erythema. Apnea may be present, regardless of encephalitis. Blood tests only show nonspecific findings. Inflammatory response and transaminase are also normal in many cases.

Britton et al reported nine infants with parechovirus encephalitis and neurodevelopmental outcomes [5]. In their study, seven of the nine infants with $\mathrm{HPeV}$ encephalitis aged $<2$ months were girls. Eight of the nine patients $(89 \%)$ had seizures, which were associated in five patients with loss of consciousness. Although $\mathrm{HPeV}$ was detected in CSF in all nine cases, CSF pleocytosis was lacking in all cases. All seven evaluated patients showed abnormal MR imaging findings and had white matter diffusion restriction. EEG anomalies were found in six of seven cases (85\%), which revealed epileptic discharges. Seven of eight patients (88\%) showed neurodevelopmental sequelae or concerns regarding abnormal neurodevelopment 12 months after discharge.

Regarding the brain MR imaging findings of $\mathrm{HPeV}$-related encephalitis, white matter high signals are seen in T2-weighted 


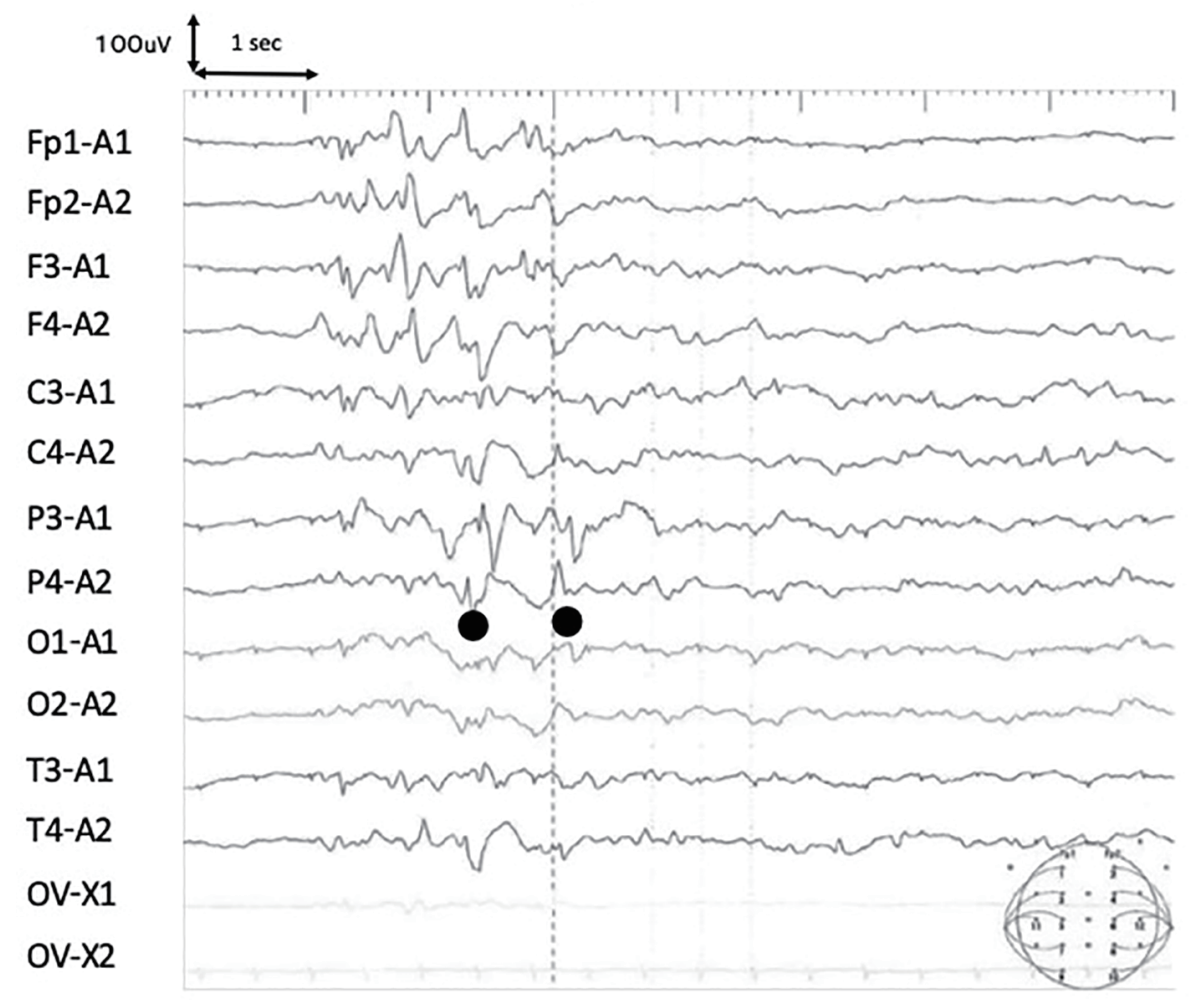

Figure 2. Electroencephalogram (EEG) on day 4 of onset. In the EEG, spike waves were independently recognized in the left and right parietal regions. Slow waves were not observed in the background activity.

images and DWIs, and the findings around the ventricle, in particular, are considered characteristic. This deficit was also observed in our case. The patient developed sepsis-like symptoms, such as fever and tachycardia, without elevated inflammatory response. She also developed complicated convulsions, respiratory disorders, neutropenia and blood clotting abnormality during the course of her case. These symptoms suggested that high cytokinemia may be responsible for elevated serum ferritin levels and high levels of IL-6. Although HPeV3-related encephalitis has usually led to neurological sequelae and death, our patient had a favorable neurological outcome.

There is no specific therapy for HPeV3 infection [6]. Supportive care for respiration and circulation is the mainstay of treatment. Fluid bolus resuscitation may be required for tachycardia and/or poor perfusion. It has also been reported that IVIG treatment was useful for HPeV1 infection [7]. All tested IVIG preparations commercially available in Japan contained high neutralizing antibody titers for HPeV3 [8]. IVIG suppressed replication of HPeV3 in vitro in a dose-dependent manner. Furthermore, early administration of IVIG was more efficient in suppressing HPeV3 RNA levels [9]. Cases of neonatal and infantile HPeV3 infection have shown serum hyperferritinemia [10]. Our patient had hyperferritinemia, high serum levels of IL-6 and cytopenia, which was assumed to reflect hypercytokinemia. Yuzurihara et al reported the cases of two neonatal patients with hemophagocytic lymphohistiocytosis-like illness, which reflected hypercytokinemia caused by $\mathrm{HPeV} 3$ and required specific therapy with corticosteroid and cyclosporine [11]. These studies support the supposition that IVIG and corticosteroid were useful treatments for our patient with $\mathrm{HPeV} 3$-related encephalitis and were associated with her favorable neurological outcome.

In conclusion, we have reported a case of a 1-month-old girl with $\mathrm{HPeV} 3$-related encephalitis. Previous reports have noted that $\mathrm{HPeV} 3$ infections with central nervous symptoms lead to high rates of long-term neurological sequelae, and we believe that careful follow-up is necessary for neurodevelopmental outcomes. HPeV should be considered as part of the differential diagnosis for neonates and infants who present septicemic symptoms without elevated inflammatory response and lack CSF pleocytosis. When a patient is suspected of having encephalitis related to $\mathrm{HPeV}$, treatment including IVIG and corticosteroid needs to be started as soon as possible to prevent neurodevelopmental sequelae.

\section{Acknowledgments}

We would like to thank Editage (www.editage.jp) for English language editing.

\section{Financial Disclosure}

The authors received no financial support for the research and/ or authorship of this article. 


\section{Conflict of Interest}

The authors declare no potential conflict of interest with respect to the authorship and/or publication of this article.

\section{Informed Consent}

Informed written consent for publication of this case was obtained from the patient's parents.

\section{Author Contributions}

W. Miura, E. Momoki, Y. Kasuga, K. Kimura and K. Fuwa provided medical care and contributed to preparation of the manuscript. W. Miura and T. Fuchigami drafted the manuscript. T. Fuchigami designed the manuscript outline and provided conceptual advice. H. Ushijima performed the HPeV3 measurement by polymerase chain reaction assay. Y. Fujita and I. Morioka supervised the entire study. All authors read and approved the final manuscript.

\section{References}

1. Aizawa Y, Izumita R, Saitoh A. Human parechovirus type 3 infection: An emerging infection in neonates and young infants. J Infect Chemother. 2017;23(7):419426.

2. Pariani E, Pellegrinelli L, Pugni L, Bini P, Perniciaro S, Bubba L, Primache V, et al. Two cases of neonatal human parechovirus 3 encephalitis. Pediatr Infect Dis J. 2014;33(11):1191-1193.

3. Sedmak G, Nix WA, Jentzen J, Haupt TE, Davis JP, Bhattacharyya S, Pallansch MA, et al. Infant deaths associated with human parechovirus infection in Wisconsin. Clin Infect Dis. 2010;50(3):357-361.

4. Miwa K, Shibayama N, Moriguchi T, Goto J, Yanagisawa M, Yamazaki Y, Jung G, et al. A rapid enzymelinked immunosorbent assay with two modes of detection for measuring cytokine concentration. J Clin Lab Anal. 2009;23(1):40-44.

5. Britton PN, Dale RC, Nissen MD, Crawford N, Elliott E, Macartney K, Khandaker G, et al. Parechovirus encephalitis and neurodevelopmental outcomes. Pediatrics. 2016;137(2):e20152848.

6. Wildenbeest JG, Harvala H, Pajkrt D, Wolthers KC. The need for treatment against human parechoviruses: how, why and when? Expert Rev Anti Infect Ther. 2010;8(12):1417-1429.

7. Wildenbeest JG, Wolthers KC, Straver B, Pajkrt D. Successful IVIG treatment of human parechovirus-associated dilated cardiomyopathy in an infant. Pediatrics. 2013;132(1):e243-247.

8. Aizawa Y, Watanabe K, Oishi T, Hirano H, Hasegawa I, Saitoh A. Role of Maternal Antibodies in Infants with Severe Diseases Related to Human Parechovirus Type 3. Emerg Infect Dis. 2015;21(11):1966-1972.

9. Izumita R, Aizawa Y, Watanabe K, Saitoh A. A role of intravenous immunoglobulin in human parechovirus type 3 infection in an in vitro model. Open Forum Infect Dis. 2016;3(suppl 1):152.

10. Hara S, Kawada J, Kawano Y, Yamashita T, Minagawa $\mathrm{H}$, Okumura N, Ito Y. Hyperferritinemia in neonatal and infantile human parechovirus-3 infection in comparison with other infectious diseases. J Infect Chemother. 2014;20(1):15-19.

11. Yuzurihara SS, Ao K, Hara T, Tanaka F, Mori M, Kikuchi N, Kai S, et al. Human parechovirus-3 infection in nine neonates and infants presenting symptoms of hemophagocytic lymphohistiocytosis. J Infect Chemother. 2013;19(1):144-148. 